\title{
Review
}

\section{Roles of small molecules in somatic cell reprogramming}

\author{
Jian-bin SU ${ }^{1,2}$, Duan-qing PEI ${ }^{1,2}$, Bao-ming $\mathrm{QIN}^{1,2, *}$ \\ ${ }^{1}$ Key Laboratory of Regenerative Biology, South China Institute for Stem Cell Biology and Regenerative Medicine, Guangzhou Insti- \\ tutes of Biomedicine and Health, Chinese Academy of Sciences, Guangzhou 510530, China; ' Guangdong Provincial Key Laboratory \\ of Stem Cells and Regenerative Medicine, South China Institute for Stem Cell Biology and Regenerative Medicine, Guangzhou Insti- \\ tutes of Biomedicine and Health, Chinese Academy of Sciences, Guangzhou 510530, China
}

\begin{abstract}
The Nobel Prize in Physiology and Medicine 2012 was awarded to Sir John B GURDON and Shinya YAMANAKA for their discovery that mature cells can be reprogrammed to become pluripotent. This event reaffirms the importance of research on cell fate plasticity and the technology progress in the stem cell field and regenerative medicine. Indeed, reprogramming technology has developed at a dazzling speed within the past 6 years, yet we are still at the early stages of understanding the mechanisms of cell fate identity. This is particularly true in the case of human induced pluripotent stem cells (iPSCs), which lack reliable standards in the evaluation of their fidelity and safety prior to their application. Along with the genetic approaches, small molecules nowadays become convenient tools for modulating endogenous protein functions and regulating key cellular processes, including the mesenchymal-to-epithelial transition, metabolism, signal transduction and epigenetics. Moreover, small molecules may affect not only the efficiency of clone formation but also the quality of the resulting cells. With increasing availability of such chemicals, we can better understand the biology of stems cells and further improve the technology of generation of stem cells.
\end{abstract}

Keywords: small molecules; induced pluripotent stem cells (iPSCs); embryonic stem cells (ESCs); reprogramming; mesenchymal-toepithelial transition (MET); epigenetics; vitamin C

Acta Pharmacologica Sinica (2013) 34: 719-724; doi: 10.1038/aps.2013.73; published online 3 Jun 2013

\section{Introduction}

As depicted by Waddington almost half a century ago, cell-fate specification during development is determined by epigenetics, which was initially thought to be static and irreversible ${ }^{[1]}$. Subsequent studies have shown that somatic cell identity can be reversed to its initial developmental state via either nuclear transfer or direct reprogramming, underscoring the fact that epigenetics in somatic cells can be dramatically manipulated ${ }^{[2]}$. In contrast to nuclear transfer, which can be made more efficient by many undefined factors in the oocyte and can be completed within one cell cycle, reprogramming with only a few embryonic stem cells (ESCs) enriched transcription factors is usually inefficient and is dependent on continuous cell division. However, this system provides a defined platform and numerous opportunities to improve stem cell technology and to decipher the mystery of cell fate maintenance. Indeed, within the past 6 years, we have witnessed how this technol-

\footnotetext{
* To whom correspondence should be addressed.

E-mail qin_baoming@gibh.ac.cn

Received 2013-03-16 Accepted 2013-05-06
}

ogy has not only paved the way for regenerative medicine but also expanded the frontiers of the stem cell field ${ }^{[3]}$.

Comparing the differences between fibroblasts (the most commonly used somatic cell type) and ESCs and investigating in detail the defined intermediate cell populations, scientists have identified some key steps and roadblocks in reprogramming. First, somatic cells usually have a limited proliferation potential, and thus, senescence needs to be overcome before the cells can exhibit their self-renewal capability, which is a hallmark feature of ESCs ${ }^{[4]}$. Second, fibroblasts differ from ESCs in their cell morphology, where the former demonstrates mesenchymal morphology, and the latter exhibits epithelial morphology. Mesenchymal-epithelial transition (MET) is required to initiate reprogramming ${ }^{[5]}$. Third, somatic cells use mitochondrial oxidative phosphorylation to generate ATP, while ESCs are mainly dependent on enhanced glycolysis to generate ATP even under normoxic conditions. This metabolic switch from mitochondrial energy to glycolysis needs to occur not only to fuel reprogramming but also to help shape the global epigenetic status ${ }^{[6]}$. Finally, compared with ESCs, somatic cells have a much more condensed epigenome, among 
which the ESC-specific genes are epigenetically silenced. Thus, derepression of the pluripotency circuit is key to iPSC generation $^{[7]}$.

Compared with genetic modulation, the use of small molecules to enhance reprogramming efficiency or quality is much easier in practice. Moreover, these molecules may serve as useful probes to pinpoint potentially important stages in this process. In this review, we will summarize the utilization of such compounds in the iPSC field, with a particular emphasis on compounds that are more effective or those whose mechanisms are better understood. In particular, we will highlight the epigenetic modulation and the relevant molecular basis of cell fate transition. Other excellent reviews of a similar topic can be found elsewhere ${ }^{[8,9]}$.

\section{Small molecules regulate the mesenchymal-to-epithelial transition}

Initially, TGF- $\beta$-receptor inhibitors, such as SB-431542 and E-616452 (or RepSox) were found to enhance both mouse and human reprogramming ${ }^{[10-12]}$. RepSox, in addition to Sox2 replacement, further induced Nanog expression ${ }^{[10]}$. Lin et al also speculated that the occurrence of the MET might be important in reprogramming ${ }^{[11]}$. Maherali and Hochedlinger further showed that TGF- $\beta$, a potent epithelial-to-mesenchymal transition (EMT) inducer, blocked reprogramming. Interestingly, the changes in cellular morphology occurred rapidly from a mesenchymal-like to an epithelial-like cell in the first few days after exogenous factor transduction. These studies suggested that the MET might be required for reprogramming. Subsequently, two parallel studies confirmed this hypothesis using different approaches ${ }^{[13,14]}$. Li et al found that the Yamanaka factors synergistically induced this transition via the indirect inhibition of the TGF- $\beta$ signaling pathway, which was constitutively activated in fibroblasts, as well as the direct activation of epithelial gene expression ${ }^{[14]}$. In addition, overexpression of E-cadherin, an epithelial marker, significantly improved and even replaced Oct4 during iPSC generation ${ }^{[15]}$. Consistent with these findings, two natural flavones, apigenin and luteolin, enhanced reprogramming efficiency via the upregulation of E-cadherin expression at the transcriptional level ${ }^{[15]}$.

\section{Small molecules regulate metabolic reprogramming and signaling pathways}

Somatic cells usually generate energy from mitochondrial oxidative phosphorylation (OXPHOS). In contrast, pluripotent stem cells enhance glycolysis to meet the high anabolic requirements ${ }^{[6,16]}$. This difference in metabolic needs has been suggested by several earlier studies. In 2009, Yoshida et al discovered that hypoxia enhanced both mouse and human reprogramming ${ }^{[17]}$. Although the detailed mechanisms were not discussed in this study, hypoxia may induce glycolysis via the stabilization and activation of the hypoxia-inducing factor $(\mathrm{HIF})^{[18]}$. In 2010, ultrastructural characterization of both mouse and human iPSCs showed that the mitochondria in these cells were nearly indistinguishable from those in ESCs but were distinct from the parental somatic cells ${ }^{[19,20]}$. These studies indicated that a metabolic switch in mitochondrial function might be a necessary step in the generation of iPSCs. In the same year, Zhu et al reported the effects of modulating metabolism using several compounds ${ }^{[2]}$. The study showed that the direct and indirect activation of glycolysis or the blockade of mitochondrial OXPHOS using a variety of compounds, such as fructose 2,6-bisphosphate (F2,6P, PFK1 activator), 2,4dinitrophenol (DNP, mitochondria decoupler), quercetin (HIF activator), and PS48 (3'-phosphoinositide-dependent kinase-1, PDK1, activator), enhanced reprogramming efficiency ${ }^{[21]}$. On the basis of these results, the authors proposed that the metabolic switch toward glycolysis was beneficial to reprogramming. It was not until 2011 that Folmes et al formally elaborated on this concept in further detail ${ }^{[22]}$. In their study, Folmes and colleagues first confirmed the metabolic switch from mitochondrial OXPHOS to glycolysis using metabolic footprinting and fingerprinting. Moreover, interference of glycolysis flux with 2-deoxyglucose (2-DG), 3-bromopyruvic acid (BrPA) and dichloroacetate (DCA) resulted in a significant reduction in reprogramming efficiency, which suggested that this glycolytic switch was necessary for effective iPSC generation. Furthermore, although c-Myc is well known to regulate glycolysis and mitochondrial function, the metabolic switch in reprogramming could occur in the absence of c-Myc ${ }^{[22]}$. However, the detailed mechanism of this switch remains unknown, and additional studies are required to further elucidate this mechanism $^{[6,23]}$.

Mouse embryonic stem cells (mESCs) can maintain longterm self-renewal in the absence of leukemia inhibitory factor (LIF) via dual inhibition of glycogen synthase kinase-3 (GSK-3 $\beta$ ) and mitogen-activated protein kinase/ERK kinase (MEK) using CHIR99021 and PD0325901, respectively (2 inhibitors, 2i). This is known as the ground state ${ }^{[24]}$. These two inhibitors have been shown to enhance reprogramming efficiency in both mouse embryonic fibroblasts (MEFs) and neural stem cells and to further facilitate reprogramming in preiPSCs ${ }^{[25]}$. Importantly, inhibition of GSK-3 $\beta$ in mESCs has been shown to induce the expression of $\operatorname{Esrrb}^{[26]}$, which was previously shown to be redundant with Klf4 in reprogramming ${ }^{[27]}$. A parallel study also showed that Esrrb could replace endogenous Nanog in both mESCs and reprogramming ${ }^{[28]}$. Interestingly, a more recent transcriptomic comparison between $2 \mathrm{i}$ and serum cultured mESCs showed a remarkable upregulation of metabolic genes in the $2 \mathrm{i}$ condition ${ }^{[29]}$. Thus, it would be interesting to investigate the potential link between $2 \mathrm{i}$ and metabolism in reprogramming.

The complexity of the regulation of the signaling pathways may be more complicated in the context of reprogramming and may at times even generate controversial conclusions. A recent kinase inhibitor screening identified new barriers of reprogramming, such as p38, inositol trisphosphate 3-kinase (IP3K) and Aurora A kinase (Aurka) ${ }^{[30]}$. However, stressmediated activation of $\mathrm{p} 38$ was shown to promote iPSC generation $^{[31]}$. An additional study also found that p53 suppression by Aurka was required for reprogramming ${ }^{[32]}$. Future 
studies of these inconsistencies may help to better understand how specific signaling pathways are coordinated in the larger context of cell fate transition.

\section{Small molecules regulate epigenetic status}

John Gurdon's success in the use of nuclear transfer demonstrated that the genetic material in somatic cells was nearly identical to that in zygotes. Moreover, epigenetic regulation determines cell identity and can be altered ${ }^{[33]}$. Epigenetic modifications mainly fall into two categories: DNA methylation and histone modification. In higher organisms, DNA methylation occurs almost exclusively in CpG dinucleotides, and non-CpG methylation appears to be strictly limited to specific developmental contexts ${ }^{[34]}$. Although DNA methylation is generally considered to be a "silencing" epigenetic mark, its relationship with gene transcription is more complicated and usually dependent on histone modifications, which are far more diverse in both type and function. Commonly occurring on highly basic histone amino $(\mathrm{N})$ tails that protrude from the nucleosomes, these modifications may change the local charge property of the histones and result in further alterations in both its structure and interactions with other proteins or DNA, which subsequently affects gene expression ${ }^{[35]}$. Among the vast number of histone modifications, acetylation and methylation are better understood, although its correlation with gene expression has only been established in a dozen histone marks. Compared to most somatic cell types, the epigenome of ESCs is more "open" and is marked by a globally lower level of DNA methylation at the CpG islands (CGI) of gene promoters, a higher level of histone acetylation and a unique pattern of histone methylation. To maintain pluripotency in ESCs, the major histone modifier polycomb group of proteins (PcGs) repress the expression of lineage-specific genes via histone methylation mainly at H3K4 and H3K37 and histone ubiquitylation at $\mathrm{H} 2 \mathrm{AK} 119^{[36]}$. Reprogramming is a derepressing process, and it is not surprising that interference with the repressive modifications usually improves iPSC generation.

In addition, methylation at the promoter CGIs is often linked to long-term repression, which can be usually found in pluripotent genes in somatic cells and is maintained by DNA methyltransferases (DNMTs). Demethylation at these loci is considered to be one of the key rate-limiting steps in reprogramming. Mikkelsen et al and Huangfu et al showed that the DNMT inhibitor, 5-AZ, improved the efficiency of mouse iPSC generation ${ }^{[37,38]}$. However, this effect was limited, and according to a more recent study, it may function more efficiently only in the late stages of reprogramming ${ }^{[39]}$. The recent intensive characterization of DNA hydroxylases (TETs) and 5-hydroxymethylcytosine (5-hmC) raises the new possibility of active DNA demethylation ${ }^{[40]}$. Indeed, according to a cell fusion-based study, both TET1 and TET2 interact with Nanog and promote reprogramming ${ }^{[41]}$, although their specific roles might differ ${ }^{[42]}$. More strikingly, TET1 could substitute for Oct4 and may generate fully competent iPSCs ${ }^{[43]}$. These discoveries have greatly expanded our vision of the dimension of DNA methylation dynamics in cell fate transition.
Histone acetylation occurs at multiple lysine residues and is generally linked to gene activation, which is derived from its effect in weakening the interaction between histones and DNA. Pluripotent genes are highly acetylated in ESCs compared to somatic cells ${ }^{[44]}$, suggesting that the inhibition of histone deacetylation may benefit the transition. Indeed, histone deacetylase inhibitors, such as valproic acid (VPA), butyrate, TSA and SAHA, have all been shown to enhance reprogramming efficiency ${ }^{[38,45-47]}$. Intriguingly, less specific VPA appears to be more potent, improves protein-reprogramming ${ }^{[48]}$ and is indispensable for microRNA-reprogramming ${ }^{[49]}$. Furthermore, it can partially rescue the abnormal imprinting of the Dlk1-Dio3 locus, which supports the development of all-iPSC mice $^{[50]}$. Interestingly, butyrate was shown to play distinct roles in different metabolic contexts ${ }^{[51]}$, which indicates that this compound might function differently at different stages of reprogramming. Butyrate can also be metabolized into acetyl-CoA, provides energy and facilitates histone acetylation during the initial stages when the metabolic switch occurs. When metabolic reprogramming is completed and endogenous acetyl-CoA is sufficient to sustain the cell's anabolic needs, butyrate may simply function as an HDAC inhibitor. Thus, butyrate may change the histone acetylation in a more gradual and smooth manner, which may explain its lower toxicity when compared to VPA in human reprogramming ${ }^{[45]}$. Recently, the histone deacetylase inhibitor SAHA, when conjugated with the specific DNA binding hairpin pyrrole-imidazole polyamides (PIPs), has been shown to induce the rapid expression of an epithelial marker and pluripotent genes in $\mathrm{MEFs}^{[52]}$. However, the effect and potential of such synthetic small molecules in reprogramming has yet to be evaluated.

As one of the most complicated histone modifications, histone methylation occurs on many lysine and, to a lesser degree, arginine residues and is regulated by methyltransferases and demethylases ${ }^{[53]}$. This more diverse pattern of methylation, compared to other types of modifications, indicates its potentially more flexible and dynamic regulatory role. Moreover, several inhibitors for methyltransferases of these repressive histone marks enhance reprogramming efficiency. For example, BIX-01294, an H3K9 methyltransferase G9a inhibitor, has been shown to enhance Oct 4 and Klf4 reprogramming and may even substitute for Oct4 ${ }^{[54,55]}$. Furthermore, EPZ004777, an H3K79 methyltransferase Dot11 inhibitor, can enhance human reprogramming ${ }^{[56]}$. In addition to these inhibitors, inactivation of demethylases on active marks may also function in a similar manner. More precisely, parnate, a histone H3K4 demethylase LSD1 inhibitor, was found to enable Oct4 and Klf4 reprogramming in human primary keratinocytes when combined with the GSK-3 $\beta$ inhibitor (CHIR99021) ${ }^{[57]}$. Similarly, LiCl, an anti-psychotic drug, facilitates one- (Oct4) or two-factor (OS or OK)-mediated reprogramming by downregulating $\mathrm{LSD}^{[58]}$.

\section{Role of vitamin $\mathbf{C}$ in reprogramming}

Taken together, these epigenetic modulating chemicals are inhibitors for enzymes that contribute to gene repression. 
Activators or agonists of histone demethylases have long been pursued; however, due to the very limited structural and functional information available, the generation of agonists that are specific for demethylases is currently extremely challenging. However, the search for broad-spectrum activators may also be a good alternative. Indeed, this was the case for the re-discovered role of vitamin $\mathrm{C}(\mathrm{Vc})$ in epigenetic reprogramming.

Vc has been initially reported to enhance the generation of both mouse and human iPSCs, but surprisingly, such an effect was independent from its traditional antioxidant properties ${ }^{[59]}$. Biochemically, Vc is a reducing co-factor for a large family of $\mathrm{Fe}^{2+}$ and a-ketoglutarate-dependent dioxygenases, among which collagen prolyl hydroxylase and HIF prolyl hydroxylase are the best studied ${ }^{[60]}$. Intriguingly, this superfamily also consists of two subgroups of enzymes that function as epigenetic modifiers, ie, jumonji-domain-containing histone lysyl and arginine demethylases and TET DNA hydroxylases ${ }^{[6] 1}$. Thus, Vc may regulate gene expression through these enzymes.

Indeed, a simple comparison of the bulk histone methylation changes in cells treated in the presence or absence of $\mathrm{Vc}$ identified a specific reduction in $\mathrm{H} 3 \mathrm{~K} 36 \mathrm{me} 2 / 3$. This resulted in the discovery of the potent role of Jhdm1a and $1 \mathrm{~b}$ in reprogramming ${ }^{[62]}$. These two enzymes demethylated H3K36me2/3 and partially mediated the enhanced effects of $\mathrm{Vc}_{\mathrm{c}}$ in iPSC generation. More strikingly, their overexpression greatly enhanced its efficiency, and Jhdm $1 b$ also helped Oct4 to achieve high efficient reprogramming in the presence of Vc. More recently, Chen et al characterized the role of Vc in converting pre-iPSCs into full-iPSCs and identified the histone mark H3K9 methylation, casted by BMP4 in serum, as the major epigenetic barrier in reprogramming using traditional methods. The jumonji-containing histone demethylases, $\mathrm{KDM} 3 / 4$, were required to mediate the effect of $\mathrm{Vc}^{[63]}$. Importantly, BMPs facilitated MET ${ }^{[13,64]}$ and could substitute for Klf4 ${ }^{[64]}$. This discrepancy might be due to BMP-activated pathways, which play different roles in different transcription factor combinations.

Apart from the efficiency aspect, Stadtfeld et al demonstrated that the addition of $\mathrm{Vc}_{\mathrm{c}}$ in the culture medium improved the quality of iPSCs in a tetraploid-complement test by maintaining adequate imprinting at the Dlk1-Dio3 locus ${ }^{[65]}$, which was previously responsible for the generation of all-iPSC mice ${ }^{[50,66]}$. In addition to DNA demethylation, these authors also found that Vc helped to maintain the active histone marks such as H3K4me3 and H3 acetylation. Moreover, it is plausible to assume that both jumonji and TET enzymes regulated this process, either independently or synergistically ${ }^{\left[{ }^{[6]}\right]}$.

Taken together, these studies not only provide evidence that Vc plays an important role in reprogramming by activating specific epigenetic modulators but also greatly expand our view of how cellular identity is maintained and manipulated (Figure 1). Thus, future studies will be necessary to clarify the mechanisms underlying Vc activation of the jumonji and TET enzymes and how it consequentially modulates cell fate.

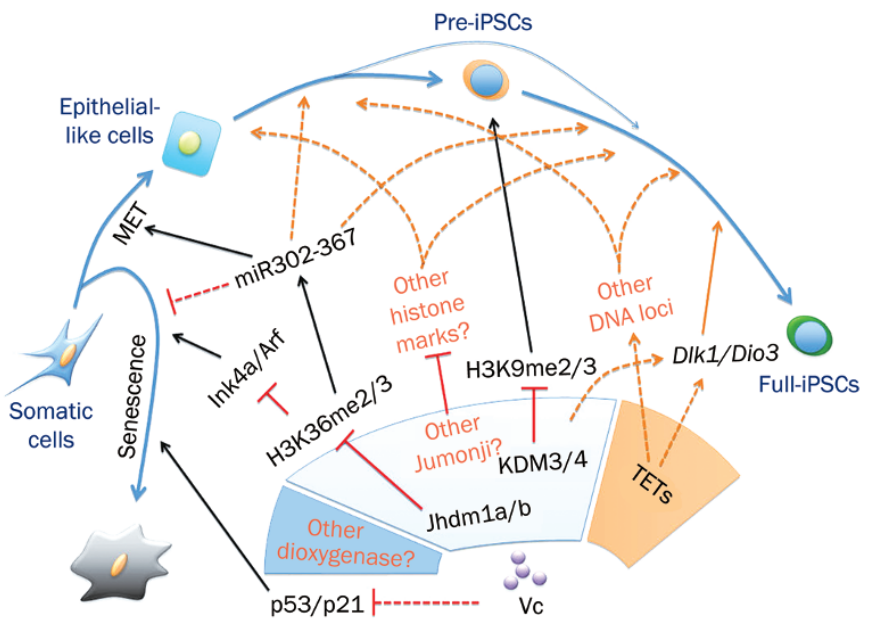

Figure 1. Vc-regulated reprogramming. The dashed lines indicate potential targets or effects.

\section{Perspectives}

Dozens of small molecules have been found to enhance reprogramming efficiency or quality (Figure 2), and many more small molecules may be identified via large-scale or candidate screening in the near future. These efforts will help to achieve one of the ultimate goals in this field: chemical-only reprogramming. From a different perspective, the precise mechanisms for most of these compounds are still unclear, and an investigation of this issue will uncover additional requirements for and barriers to somatic cell reprogramming. These studies might also be highly valuable for advancing the technology of other cell fate transitions, such as the differentiation ${ }^{[68]}$, transdifferentiation ${ }^{[69]}$ and maintenance of somatic stem cells in vitro ${ }^{[70]}$.

\section{Acknowledgements}

We thank Miguel Angel ESTEBAN, Qiang ZHUANG, and $\mathrm{Xi}$-chen $\mathrm{BAO}$ for their helpful suggestions. The studies in the authors' laboratories were supported by the Strategic Priority Research Program of the Chinese Academy of Sciences (grant XDA01020401) and the National Basic Research Program of China (grants 2011CBA01106 and 2011CBA01004).

\section{References}

1 Waddington $\mathrm{CH}$. The strategy of the genes. London: George Allen and Unwin; 1957.

2 Jaenisch R. Nuclear cloning and direct reprogramming: the long and the short path to Stockholm. Cell Stem Cell 2012; 11: 744-7.

3 Yamanaka S. Induced pluripotent stem cells: past, present, and future. Cell Stem Cell 2012; 10: 678-84.

4 Banito A, Gil J. Induced pluripotent stem cells and senescence: learning the biology to improve the technology. EMBO Rep 2010; 11: 353-9.

5 Esteban MA, Bao X, Zhuang Q, Zhou T, Qin B, Pei D. The mesenchymal-to-epithelial transition in somatic cell reprogramming. Curr Opin Genet Dev 2012; 22: 423-8.

6 Zhang J, Nuebel E, Daley GQ, Koehler CM, Teitell MA. Metabolic 


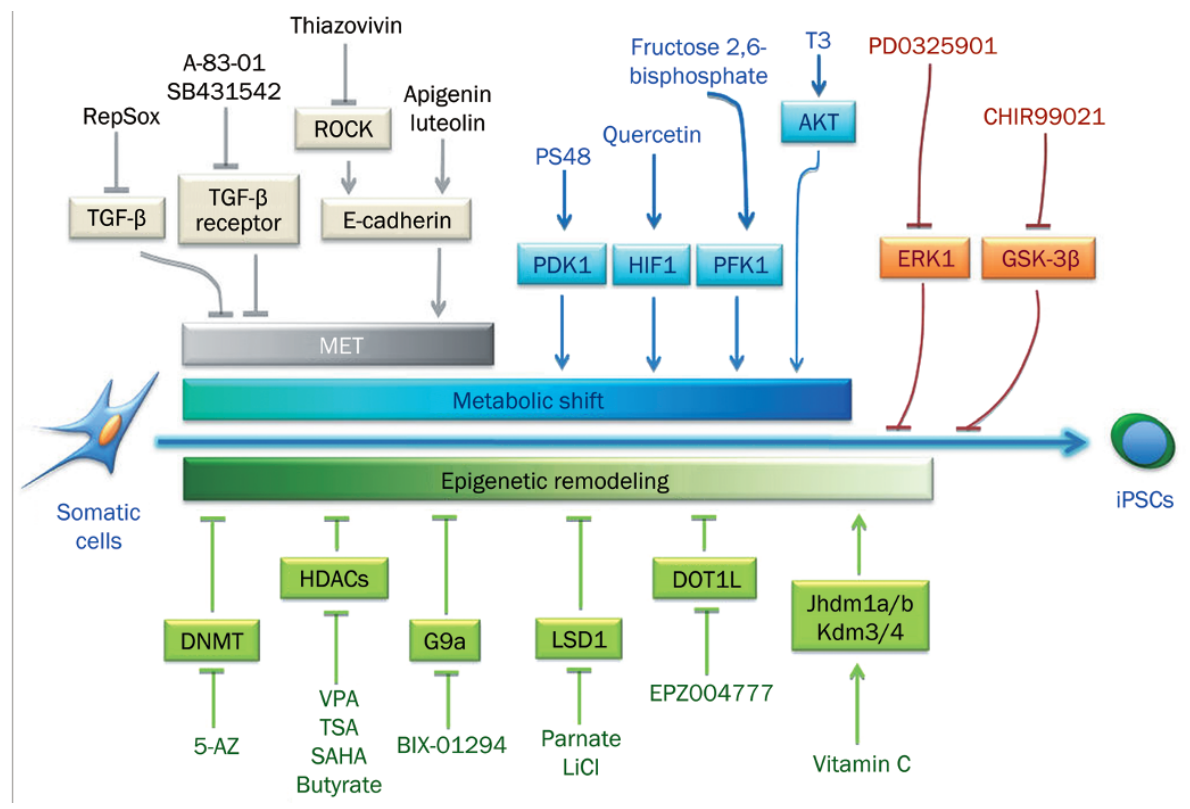

Figure 2. Small molecule-regulated reprogramming. The direct or potential targets and the related processes are listed in different colored groups. The dashed lines indicate indirect or potential targeting.

regulation in pluripotent stem cells during reprogramming and selfrenewal. Cell Stem Cell 2012; 11: 589-95.

7 Pasque V, Jullien J, Miyamoto K, Halley-Stott RP, Gurdon JB. Epigenetic factors influencing resistance to nuclear reprogramming. Trends Genet 2011; 27: 516-25.

8 Nie B, Wang $\mathrm{H}$, Laurent T, Ding S. Cellular reprogramming: a small molecule perspective. Curr Opin Cell Biol 2012; 24: 784-92.

9 Li W, Jiang K, Ding S. Concise review: A chemical approach to control cell fate and function. Stem Cells 2012; 30: 61-8.

10 Ichida JK, Blanchard J, Lam K, Son EY, Chung JE, Egli D, et al. A small-molecule inhibitor of TGF-beta signaling replaces sox 2 in reprogramming by inducing nanog. Cell Stem Cell 2009; 5: 491-503.

11 Lin T, Ambasudhan R, Yuan X, Li W, Hilcove S, Abujarour R, et al. A chemical platform for improved induction of human iPSCs. Nat Methods 2009; 6: 805-8.

12 Maherali N, Hochedlinger K. TGFbeta signal inhibition cooperates in the induction of iPSCs and replaces Sox2 and cMyc. Curr Biol 2009; 19: 1718-23.

13 Samavarchi-Tehrani P, Golipour A, David L, Sung HK, Beyer TA, Datti $A$, et al. Functional genomics reveals a BMP-driven mesenchymal-toepithelial transition in the initiation of somatic cell reprogramming. Cell Stem Cell 2010; 7: 64-77.

14 Li R, Liang J, Ni S, Zhou T, Qing X, Li H, et al. A mesenchymal-toepithelial transition initiates and is required for the nuclear reprogramming of mouse fibroblasts. Cell Stem Cell 2010; 7: 51-63.

15 Chen T, Yuan D, Wei B, Jiang J, Kang J, Ling K, et al. E-cadherinmediated cell-cell contact is critical for induced pluripotent stem cell generation. Stem Cells 2010; 28: 1315-25.

16 Kondoh H, Lleonart ME, Nakashima Y, Yokode M, Tanaka M, Bernard $\mathrm{D}$, et al. A high glycolytic flux supports the proliferative potential of murine embryonic stem cells. Antioxid Redox Signal 2007; 9: 293-9.

17 Yoshida Y, Takahashi K, Okita K, Ichisaka T, Yamanaka S. Hypoxia enhances the generation of induced pluripotent stem cells. Cell Stem Cell 2009; 5: 237-41.

18 Majmundar AJ, Wong WJ, Simon MC. Hypoxia-inducible factors and the response to hypoxic stress. Mol Cell 2010; 40: 294-309.

19 Zeuschner D, Mildner K, Zaehres H, Scholer HR. Induced pluripotent stem cells at nanoscale. Stem Cells Dev 2010; 19: 615-20.

20 Prigione A, Fauler B, Lurz R, Lehrach H, Adjaye J. The senescencerelated mitochondrial/oxidative stress pathway is repressed in human induced pluripotent stem cells. Stem Cells 2010; 28: 721-33.

21 Zhu S, Li W, Zhou H, Wei W, Ambasudhan R, Lin T, et al. Reprogramming of human primary somatic cells by OCT4 and chemical compounds. Cell Stem Cell 2010; 7: 651-5.

22 Folmes CD, Nelson TJ, Martinez-Fernandez A, Arrell DK, Lindor JZ, Dzeja PP, et al. Somatic oxidative bioenergetics transitions into pluripotency-dependent glycolysis to facilitate nuclear reprogramming. Cell Metab 2011; 14: 264-71.

23 Folmes CD, Dzeja PP, Nelson TJ, Terzic A. Metabolic plasticity in stem cell homeostasis and differentiation. Cell Stem Cell 2012; 11: 596606.

24 Ying QL, Wray J, Nichols J, Batlle-Morera L, Doble B, Woodgett J, et al. The ground state of embryonic stem cell self-renewal. Nature 2008; 453: 519-23.

25 Silva J, Barrandon O, Nichols J, Kawaguchi J, Theunissen TW, Smith A. Promotion of reprogramming to ground state pluripotency by signal inhibition. PLoS Biol 2008; 6: e253.

26 Martello G, Sugimoto T, Diamanti E, Joshi A, Hannah R, Ohtsuka S, et al. Esrrb is a pivotal target of the Gsk3/Tcf3 axis regulating embryonic stem cell self-renewal. Cell Stem Cell 2012; 11: 491-504.

27 Feng B, Jiang J, Kraus P, Ng JH, Heng JC, Chan YS, et al. Reprogramming of fibroblasts into induced pluripotent stem cells with orphan nuclear receptor Esrrb. Nat Cell Biol 2009; 11: 197-203.

28 Festuccia N, Osorno R, Halbritter F, Karwacki-Neisius V, Navarro P, Colby $\mathrm{D}$, et al. Esrrb is a direct Nanog target gene that can substitute for Nanog function in pluripotent cells. Cell Stem Cell 2012; 11: 47790.

29 Marks H, Kalkan T, Menafra R, Denissov S, Jones K, Hofemeister H, et al. The transcriptional and epigenomic foundations of ground state pluripotency. Cell 2012; 149: 590-604. 
mouse induced pluripotent stem cells. Nature 2010; 465: 175-81.

30 Li Z, Rana TM. A kinase inhibitor screen identifies small-molecule enhancers of reprogramming and iPS cell generation. Nat Commun 2012; 3: 1085-95.

31 Xu X, Wang Q, Long Y, Zhang R, Wei X, Xing M, et al. Stress-mediated p38 activation promotes somatic cell reprogramming. Cell Res 2013; 23: 131-41.

32 Lee DF, Su J, Ang YS, Carvajal-Vergara X, Mulero-Navarro S, Pereira $\mathrm{CF}$, et al. Regulation of embryonic and induced pluripotency by aurora kinase-p53 signaling. Cell Stem Cell 2012; 11: 179-94.

33 Gurdon JB, Elsdale TR, Fischberg M. Sexually mature individuals of Xenopus laevis from the transplantation of single somatic nuclei. Nature 1958; 182: 64-5.

34 Jones PA. Functions of DNA methylation: islands, start sites, gene bodies and beyond. Nat Rev Genet 2012; 13: 484-92.

35 Bannister AJ, Kouzarides T. Regulation of chromatin by histone modifications. Cell Res 2011; 21: 381-95.

36 Han JW, Yoon YS. Epigenetic landscape of pluripotent stem cells. Antioxid Redox Signal 2012; 17: 205-23.

37 Mikkelsen TS, Hanna J, Zhang X, Ku M, Wernig M, Schorderet P, et al. Dissecting direct reprogramming through integrative genomic analysis. Nature 2008; 454: 49-55.

38 Huangfu D, Maehr R, Guo W, Eijkelenboom A, Snitow M, Chen AE, et al. Induction of pluripotent stem cells by defined factors is greatly improved by small-molecule compounds. Nat Biotechnol 2008; 26: 795-7.

39 Polo JM, Anderssen E, Walsh RM, Schwarz BA, Nefzger CM, Lim SM, et al. A molecular roadmap of reprogramming somatic cells into iPS cells. Cell 2012; 151: 1617-32.

40 Branco MR, Ficz G, Reik W. Uncovering the role of 5-hydroxymethylcytosine in the epigenome. Nat Rev Genet 2012; 13: 7-13.

41 Costa Y, Ding J, Theunissen TW, Faiola F, Hore TA, Shliaha PV, et al. Nanog-dependent function of TET1 and TET2 in establishment of pluripotency. Nature 2013; 495: 370-4.

42 Piccolo FM, Bagci H, Brown KE, Landeira D, Soza-Ried J, Feytout A, et al. Different roles for Tet1 and Tet2 proteins in reprogrammingmediated erasure of imprints induced by EGC fusion. Mol Cell 2013; 49: 1023-33.

43 Gao Y, Chen J, Li K, Wu T, Huang B, Liu W, et al. Replacement of Oct4 by Tet1 during iPSC induction reveals an important role of DNA methylation and hydroxymethylation in reprogramming. Cell Stem Cell 2013; 12: 453-69.

44 Kimura H, Tada M, Nakatsuji N, Tada T. Histone code modifications on pluripotential nuclei of reprogrammed somatic cells. Mol Cell Biol 2004; 24: 5710-20.

45 Mali P, Chou BK, Yen J, Ye Z, Zou J, Dowey S, et al. Butyrate greatly enhances derivation of human induced pluripotent stem cells by promoting epigenetic remodeling and the expression of pluripotencyassociated genes. Stem Cells 2010; 28: 713-20.

46 Liang G, Taranova O, Xia K, Zhang Y. Butyrate promotes induced pluripotent stem cell generation. J Biol Chem 2010; 285: 25516-21.

47 Huangfu D, Osafune K, Maehr R, Guo W, Eijkelenboom A, Chen S, et al. Induction of pluripotent stem cells from primary human fibroblasts with only Oct4 and Sox2. Nat Biotechnol 2008; 26: 1269-75.

48 Zhou H, Wu S, Joo JY, Zhu S, Han DW, Lin T, et al. Generation of induced pluripotent stem cells using recombinant proteins. Cell Stem Cell 2009; 4: 381-4.

49 Anokye-Danso F, Trivedi CM, Juhr D, Gupta M, Cui Z, Tian Y, et al. Highly efficient miRNA-mediated reprogramming of mouse and human somatic cells to pluripotency. Cell Stem Cell 2011; 8: 376-88.

50 Stadtfeld M, Apostolou E, Akutsu H, Fukuda A, Follett P, Natesan S, et al. Aberrant silencing of imprinted genes on chromosome $12 \mathrm{qF} 1$ in
51 Donohoe DR, Collins LB, Wali A, Bigler R, Sun W, Bultman SJ. The warburg effect dictates the mechanism of butyrate-mediated histone acetylation and cell proliferation. Mol Cell 2012; 48: 612-26.

52 Pandian GN, Nakano Y, Sato S, Morinaga H, Bando T, Nagase H, et al. A synthetic small molecule for rapid induction of multiple pluripotency genes in mouse embryonic fibroblasts. Sci Rep 2012; 2: 544-51.

53 Greer EL, Shi Y. Histone methylation: a dynamic mark in health, disease and inheritance. Nat Rev Genet 2012; 13: 343-57.

54 Shi Y, Desponts C, Do JT, Hahm HS, Scholer HR, Ding S. Induction of pluripotent stem cells from mouse embryonic fibroblasts by Oct4 and KIf4 with small-molecule compounds. Cell Stem Cell 2008; 3: 568-74.

55 Shi Y, Do JT, Desponts C, Hahm HS, Scholer HR, Ding S. A combined chemical and genetic approach for the generation of induced pluripotent stem cells. Cell Stem Cell 2008; 2: 525-8.

56 Onder TT, Kara N, Cherry A, Sinha AU, Zhu N, Bernt KM, et al. Chromatin-modifying enzymes as modulators of reprogramming. Nature 2012; 483: 598-602.

57 Li W, Zhou H, Abujarour R, Zhu S, Young Joo J, Lin T, et al. Generation of human-induced pluripotent stem cells in the absence of exogenous Sox2. Stem Cells 2009; 27: 2992-3000.

58 Wang Q, Xu X, Li J, Liu J, Gu H, Zhang R, et al. Lithium, an antipsychotic drug, greatly enhances the generation of induced pluripotent stem cells. Cell Res 2011; 21: 1424-35.

59 Esteban MA, Wang T, Qin B, Yang J, Qin D, Cai J, et al. Vitamin C enhances the generation of mouse and human induced pluripotent stem cells. Cell Stem Cell 2010; 6: 71-9.

60 Horton JR, Upadhyay AK, Qi HH, Zhang X, Shi Y, Cheng X. Enzymatic and structural insights for substrate specificity of a family of jumonji histone lysine demethylases. Nat Struct Mol Biol 2010; 17: 38-43.

61 Loenarz C, Schofield CJ. Expanding chemical biology of 2-oxoglutarate oxygenases. Nat Chem Biol 2008; 4: 152-6.

62 Wang T, Chen K, Zeng X, Yang J, Wu Y, Shi X, et al. The histone demethylases Jhdm $1 \mathrm{a} / 1 \mathrm{~b}$ enhance somatic cell reprogramming in a vitamin-C-dependent manner. Cell Stem Cell 2011; 9: 575-87.

63 Chen J, Liu H, Liu J, Qi J, Wei B, Yang J, et al. H3K9 methylation is a barrier during somatic cell reprogramming into iPSCs. Nat Genet 2012; 45: 34-42.

64 Chen J, Liu J, Yang J, Chen Y, Chen J, Ni S, et al. BMPs functionally replace KIf4 and support efficient reprogramming of mouse fibroblasts by Oct4 alone. Cell Res 2011; 21: 205-12.

65 Stadtfeld M, Apostolou E, Ferrari F, Choi J, Walsh RM, Chen T, et al. Ascorbic acid prevents loss of DIk1-Dio3 imprinting and facilitates generation of all-iPS cell mice from terminally differentiated $B$ cells. Nat Genet 2012; 44: 398-405, S1-2.

66 Liu L. Activation of the imprinted DIk1-Dio3 region correlates with pluripotency levels of mouse stem cells. J Biol Chem 2010; 285: 19483-90.

67 Esteban MA, Pei D. Vitamin C improves the quality of somatic cell reprogramming. Nat Genet 2012; 44: 366-7.

68 Tohyama S, Hattori F, Sano M, Hishiki T, Nagahata Y, Matsuura T, et al. Distinct metabolic flow enables large-scale purification of mouse and human pluripotent stem cell-derived cardiomyocytes. Cell Stem Cell 2013; 12: 127-37.

69 Wang L, Wang L, Huang W, Su H, Xue Y, Su Z, et al. Generation of integration-free neural progenitor cells from cells in human urine. Nat Methods 2012; 10: 84-9.

70 Huang J, Nguyen-McCarty M, Hexner EO, Danet-Desnoyers G, Klein PS. Maintenance of hematopoietic stem cells through regulation of Wnt and mTOR pathways. Nat Med 2012; 18: 1778-85. 